\title{
Cherenkov Telescope Array: Unveiling the Gamma Ray Universe and its Cosmic Particle Accelerators
}

\author{
Elisabete M. de Gouveia Dal Pino \\ (on behalf of the CTA Collaboration) ${ }^{1}$ \\ ${ }^{1}$ IAG, Universidade de São Paulo, \\ Rua do Matão 1226, São Paulo, SP, Brazil \\ email: dalpino@iag.usp.br
}

\begin{abstract}
Gamma-ray astronomy has a huge potential in astrophysics, particle physics and cosmology. The Cherenkov Telescope Array (CTA) is an international initiative to build the nextgeneration ground-based gamma-ray observatory which will have a factor of 5-10 improvement in sensitivity in the $100 \mathrm{GeV}-10 \mathrm{TeV}$ range and an extension to energies well below $100 \mathrm{GeV}$ and above $100 \mathrm{TeV}$. CTA is planned to consist of two arrays (one in the North and another in the South Hemisphere) and will provide the deepest insight ever reached into the non-thermal high-energy Universe and its particle accelerators.
\end{abstract}

Keywords. instrumentation: miscellaneous, gamma-rays, acceleration of particles

\section{Overview}

Ground-based gamma-ray astronomy is a young field with enormous scientific potential. The possibility of astrophysical measurements at tera-electronvolt ( $\mathrm{TeV}$ ) energies was demonstrated in 1989 with the detection of a signal from the Crab (Pulsar Wind) Nebula above $1 \mathrm{TeV}$ with the Whipple Imaging Atmospheric Cherenkov Telescope (IACT). Since then, the instrumentation for astronomy with IACTs have evolved to the extent that a flourishing new scientific discipline has been established, with the detection of 150 sources and a major impact in astrophysics and more widely in physics. The current major arrays of IACTs (H.E.S.S., MAGIC, and VERITAS) have demonstrated the huge potential at these energies as well as the maturity of the detection technique. Many astrophysical source classes have been established, but there are indications that the known sources represent the tip of the iceberg both in terms of individual objects and source classes. The Cherenkov Telescope Array (CTA) will transform our understanding of the high-energy universe (see Actis et al.(2011) and Acharya et al.(2013) for reviews).

CTA will explore our Universe in depth in Very High Energy (VHE, E > $10 \mathrm{GeV}$ ) gamma-rays and investigate cosmic non-thermal processes, in close cooperation with observatories operating at other wavelength ranges of the electromagnetic spectrum, and those using other messengers such as cosmic rays, neutrinos and gravitational waves.

Besides anticipated high-energy astrophysics results, CTA will have a large discovery potential in key areas of astrophysics, cosmology and fundamental physics research. These include the study of the origin of cosmic rays and their impact on the constituents of the Universe, the investigation of the nature and variety of astrophysical accelerators including supernovae, neutron stars, merging stars, black holes, relativistic jets and explosions, and the inquiry into the ultimate nature of matter and physics beyond the Standard Model, searching for dark matter and effects of quantum gravity.

The major questions that CTA will address can be grouped into three broad themes: 
(1) Understanding the Origin and Role of Relativistic Cosmic Particles:

What are the sites of high-energy particle acceleration in the universe? What are the mechanisms for cosmic particle acceleration? What role do accelerated particles play in feedback on star formation and galaxy evolution?

(2) Probing Extreme Environments:

What physical processes are at work close to neutron stars and black holes? What are the characteristics of relativistic jets, winds and explosions? How intense are the radiation and the magnetic fields in cosmic voids, and how do these evolve over cosmic time?

(3) Exploring Frontiers in Physics:

What is the nature of Dark Matter? How is it distributed? Are there quantum gravitational effects on photon propagation? Do axion-like particles exist?

CTA is planned to consist of two arrays, one in the South and another in the North, aiming to: (a) increase the sensitivity level of current instruments by a factor $\sim 10$ at $1 \mathrm{TeV}$; (b) boost significantly the detection area and hence the photon rate, providing access to the shortest timescale (transient) phenomena; (c) improve substantially the angular resolution and field of view and hence the ability to image extended sources, (d) provide energy coverage for photons from $20 \mathrm{GeV}$ to at least $300 \mathrm{TeV}$, allowing the reach of high-redshifts and extreme accelerators; (e) enhance dramatically the surveying and monitoring capabilities, and the flexibility of operation, allowing for simultaneous observations of objects in multiple fields; and (f) provide access to the whole sky with sites in the two Hemispheres.

CTA will be operated as a proposal-driven open observatory, with a Science Data Centre providing access to data, analysis tools and user training. The main site will be in the southern hemisphere (in Chile), given the wealth of sources in the central region of our Galaxy and the richness of their morphological features. The northern site (in the Canary Islands) will be complementary and primarily devoted to the study of Active Galactic Nuclei (AGN), galaxy and star formation cosmological evolution, the extragalactic background light absorption (EBL), and the intergalactic magnetic fields.

The very wide energy range covered by CTA South will require the use of around 100 telescopes with at least three different sizes: large size telescopes (LSTs) with diameters of $\sim 23 \mathrm{~m}$, middle size telescopes (MSTs) with diameters of $\sim 12 \mathrm{~m}$, and small size telescopes (SSTs) with diameters $\sim 4$ to $6 \mathrm{~m}$. In the North, a focus on extragalactic science is envisaged and hence (due to gamma-gamma absorption on Mpc scales) only limited sensitivity is required. The northern instrument can therefore be implemented with only LSTs and MSTs.

There are several strong motivations for the wide CTA energy range: the lowest energies provide access to the whole universe (avoiding significant gamma-gamma absorption on the EBL), while the highest energies are needed to study the extreme accelerators which are present in our Galaxy. Besides, a wide energy range maximises the chances of serendipitous detection of new source classes with unknown spectral characteristics, for example in the search for Dark Matter with an unknown WIMP mass.

Full sky coverage ensures that extremely rare but critically important events (for example a Galactic supernova explosion, bright gravitational wave transient, or very nearby gamma-ray burst) will be accessible to CTA.

CTA represents a genuinely world-wide effort, involving institutions of 32 countries in 5 continents. Currently, the collaboration is in the prototype development phase. A Mini-Array essentially constituted of 9 dual-mirror small size telescopes (named ASTRI), is under development and its installation in the South site - as a precursor of the CTA is intended in the beginning of 2017. The full array should be deployed around 2020 
(see http://portal.cta-observatory.org/Pages/Home.aspx to access most of the special reviews on the CTA and its vast science case).

\section{Acknowledgements}

We gratefully acknowledge support from the agencies and organizations listed under Funding Agencies at this website: http://www. cta-observatory.org/.

\section{References}

Actis, M., Agnetta, G., Aharonian, F., et al. 2011, Experimental Astronomy, 32, 193

Acharya, B. S., Actis, M., Aghajani, T., et al. 2013, Astroparticle Physics, 43, 3 